\title{
Adding a subsidiary sprint audit dataset to a continuous national clinical audit - lessons from the National Hip Fracture Database
}

\author{
Authors: Meghan Liddicoat, Antony Johansen, Christopher Boulton and James Hannaford
}

\begin{abstract}
Aims
To gather patient-level clinical and facilities-level operational information on the organisation, quality and intensity of physiotherapy across England and Wales.
\end{abstract}

\section{Methods}

National clinical audits can take several years to reach the point of overwhelming recruitment, majority and reliability. At this point the audit data feeds routinely and accurately into clinical governance structures and drives improvement. It is possible, once well established, to successfully add subsidiary datasets short term for high impact sprint data collection to explore specific clinical questions.

The National Hip Fracture Database (NHFD) is a national audit which examines and improves the multidisciplinary care provided to frail and older patients. In 2017 the Chartered Society of Physiotherapy (CSP) commissioned the Royal College of Physicians (RCP) to manage the Physiotherapy Hip Fracture Sprint Audit (PHFSA) as an extension to the routine data collection of the NHFD.

NHFD captures data on every patient with hip fracture over 60 years of age presenting to an acute hospital in the NHS. PHFSA set out to extend this dataset with details of the physiotherapy and rehabilitation provided to each patient. The datasets for the PHFSA were designed by a multidisciplinary group of highly qualified clinical professionals including orthopaedics, orthogeriatrics, acute and community physiotherapy and patient representation.

In the spring of 2017 the CSP led the rapid recruitment of physiotherapists who gathered prospective data for the first 7 days in each of the three stages of the rehabilitation pathway: the acute ward, any post-acute inpatient rehabilitation setting and in community rehabilitation services. Patients who first presented between 1 May and 30 June 2017 were included.

\section{Results}

In total, 305 sites took part in PHFSA, the largest ever prospective audit of UK physiotherapy services. 177 acute hospitals admitted hip fracture patients acutely - 116 (two-thirds) of these provided data on a total of over 8,000 patients, two-thirds of all those who presented during the PHFSA acute patient recruitment phase. In addition, 142 non-acute rehabilitation/community sites across England and Wales took part - $50 \%$ of therapists were based in acute sites and $50 \%$ in rehabilitation wards or community services. The PHFSA and NHFD shared a 120-day follow-up dataset, so that only one follow-up contact was required per patient.

There were several key reasons for the success of the PHFSA; an effective collaborative relationship between the CSP and the RCP, the use of experienced hip fracture audit staff and clinical leads, the use of well-established clinical leads and staff within the hospitals and the FFFAP/NHFD brand and reputation. Planning of the project involved experts in the field of physiotherapy, acute hip fracture management, clinical audit methodology, and quality improvement (RCP). This collaboration allowed for the development of an extensive target audience contact database as well as the selection of appropriate clinical questions. Lastly, the target audience clearly displayed a desire for change. This was evidenced through the audit recruitment and uptake and through the data itself, where it is easy to see that, despite a severe lack of resourcing, physiotherapists are prioritising and thus optimising patient care and outcomes.

\section{Conclusion}

The inclusion of temporary PHFSA questions in the continuous NHFD dataset allowed detailed clinical information to be captured using the structures of a well-established national clinical audit. This was largely due to good communication between the commissioner (CSP) and the project management team (RCP), an exceptional member contact database, key clinical leads 'on the ground' and a desire for change within the clinical audience.

\section{Conflict of interest statement}

None declared. 